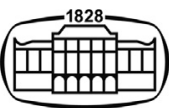

AKADÉMIAI KIADÓ

\title{
Is MDD the right target for early-stage psychedelic-assisted therapy trials?
}

\author{
BENJAMIN R. LEWIS* (0) and KEVIN BYRNE
}

\section{Journal of Psychedelic Studies}

5 (2021) 2, 65-68

DOI:

10.1556/2054.2021.00180

(C) 2021 The Author(s)

\section{REVIEW ARTICLE}

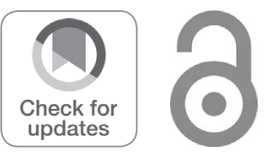

*Corresponding author. Tel.: +1 (435) 6555128.

E-mail: ben.lewis@hsc.utah.edu
Huntsman Mental Health Institute, University of Utah, 501 Chipeta Way, Salt Lake City, UT 84108, USA

Received: May 4, 2021 • Revised manuscript received: August 5, 2021 • Accepted: August 12, 2021 Published online: September 14, 2021

\begin{abstract}
The recently published Imperial College study of a Phase II, double-blind, randomized, controlled trial comparing psilocybin-assisted therapy to a six-week titration of escitalopram for Major Depressive Disorder (MDD) should raise concerns for this illness category as a target of early psychedelic research given a goal of FDA approval. There are three reasons why MDD is the wrong target at this stage of research development. Firstly, the psychiatric category of MDD is heterogeneous, vaguely-defined, and overdiagnosed in a way that will problematize finding a reliable signal with psychedelic interventions (or any intervention), particularly within non-severe cases. Secondly, current rating scales for MDD (QIDS used in the Imperial College trial, but also HAM-D) are limited in approximating the kinds of things we ultimately care most about with depressive states, namely functional status, quality of life, and well-being: measures that seem more salient for psychedelic interventions and which are not adequately captured by these rating scales used in a majority of clinical trials. And thirdly, there are inherent conflicts between psychiatric conceptualizations of MDD (and its symptom amelioration) and the kinds of perspectives on one's suffering often occasioned by psychedelic experiences themselves: while these kinds of psychedelic-catalyzed openings may lead to a form of acceptance or equanimity with regards to one's life circumstances this could be in many ways orthogonal to reductions in HAM-D scores. We argue that for these reasons MDD is a non-ideal target at this stage of the science and propose alternative directions.
\end{abstract}

\section{KEYWORDS}

psilocybin, psychedelic-assisted therapy, major depressive disorder (MDD), antidepressant

We are currently witnessing a renaissance in psychedelic research. Recent work in psychiatry has been remarkably promising with a large magnitude therapeutic effects after even single drug administrations, particularly for existential distress or anxiety in cancer patients (Grffiths, 2015; Grob, Bossis, \& Griffiths, 2013), smoking cessation (Johnson, Garcia-Romeu, Cosimano, Griffiths, 2014; Johnson, Garcia-Romeu, Griffiths, 2017), and treatment-resistant depression (Carhart Harris et al., 2018). As of this writing there are currently 64 psilocybin clinical trials in various stages registered with the FDA addressing a range of psychiatric diagnoses including MDD, OCD, anorexia nervosa, alcohol use disorder, and migraine headaches (National Institutes of Health, accessed 2021). Major Depressive Disorder (MDD) has emerged as a primary target of psychedelic-assisted therapy studies, and is the disorder best positioned at present to achieve FDA approval for psilocybin administration following upcoming Phase III trials initiated by COMPASS Pathways and Usona. Research into clinical applications of classical psychedelics-understood as a class of compounds whose pharmacological action is via serotonin-2A receptor agonism-is an uphill battle given their DEA Schedule 1 status, their colored history in the 1960s counter-cultural movement, and ways in which the model of clinical application diverges from standard psychiatric practices. While there has been a recent shift in attitude towards psychedelics as possible clinical tools, the science is still in a nascent stage and at this point very vulnerable to large setbacks. One reason among several for this possible outcome would be lackluster results from clinical trials due to targeting the wrong illness categories. There is logic to pinning hopes of FDA approval and general advancement of psychedelic science on MDD: this diagnosis is shared by over 
250 million people globally and is the largest contributor to the estimated 1 billion people with disability related to mental illness. Major Depressive Disorder is increasing in frequency, associated with significant morbidity, contributes significantly to the overall disability burden of mental illness in westernized countries, and currently available treatments are limited in achieving long-term remission. However there are reasons to believe that this may not be the ideal target for psychedelic-assisted therapy trials at this stage.

The Imperial College group led by Robin Carhart-Harris recently published a phase 2, double-blind, randomized, controlled trial comparing psilocybin-assisted therapy to a six-week titration of escitalopram for MDD in the New England Journal of Medicine (Carhart-Harris et al., 2021). A total of 59 patients were enrolled with 30 assigned to the psilocybin group (which involved $2 \times 25 \mathrm{mg}$ psilocybin sessions) and 29 to the escitalopram titration group (10 mg $\times 3$ weeks then $20 \mathrm{mg} \times 3$ weeks) with the QIDS-SR-16 as the primary outcome measure. After 6 weeks, there were no significant differences between the two groups on the QIDSSR-16 (a self-report measure of depressive symptoms) however secondary measures did favor psilocybin over escitalopram. While this study represents an important step forward towards Phase 3 trials and FDA approval, these results are underwhelming and should raise concerns about this particular target for psychedelic-assisted therapy trials, or at least the methodology used in approaching this target.

While methodological changes may improve the situation, there are three reasons that MDD is nonetheless a suboptimal initial target for these interventions. To be clear, this argument hinges on the current state of the research: targets that don't make pragmatic sense at this point certainly may become indications as the science develops and the techniques and dosing strategies are further developed. Firstly, the psychiatric category of MDD is heterogeneous, vaguelydefined, and overdiagnosed in a way that will problematize finding a reliable signal with psychedelic interventions (or any intervention), particularly within non-severe cases. Secondly, current rating scales for MDD (QIDS used in the Imperial College trial, but also HAM-D) are limited in approximating the kinds of things we ultimately care most about with depressive states, namely functional status, quality of life, and well-being: measures that seem more salient for psychedelic interventions and which are not adequately captured by these rating scales used in a majority of clinical trials. And thirdly, there are inherent conflicts between psychiatric conceptualizations of MDD (and its symptom amelioration) and the kinds of perspectives on one's suffering often occasioned by psychedelic experiences themselves: while these kinds of psychedelic-catalyzed openings may lead to a form of acceptance or equanimity with regards to one's life circumstances this could be in many ways orthogonal to reductions in HAM-D scores. In other words, there may well be significant benefits to be gained with psychedelic-assisted therapies that are not wellcharacterized within standard psychiatric assessment or captured by existing tools of evaluation and rating scales. After exploring these three reasons, my commentary will explore strategies that may benefit future trials targeting MDD as well as suggest more pragmatic targets for earlystage psychedelic clinical trials with an emphasis on conditions with clearly measurable and quantifiable behavioral correlates such as substance use disorders, OCD, eating disorders, and somatization/functional neurological disorders.

\section{NOSOLOGICAL ISSUES}

Returning to the recently published Carhart-Harris study, this apparent equivalency in interventions might initially appear promising given only two medication administrations in the psilocybin group compared to daily (and presumably protracted) escitalopram administration in the SSRI group. However it is important to consider the relative effect size of SSRI interventions in this population (moderate MDD) in general: there is reason to believe that SSRIs are effective in cases of severe MDD however in mild to moderate illness there is limited evidence that SSRIs separate from placebo (Jakobsen, Gluud, \& Kirsch, 2020; Kirsch, 2019). STAR-D results suggest that only approximately $30 \%$ of individuals will achieve remission with a SSRI trial that is adequate in terms of dose and duration (Howland, 2008). Despite increasing rates of pharmacological treatment (10$13 \%$ of U.S. adults are currently taking antidepressant medications (Brody and $\mathrm{Gu}, 2020$ ), incidence of MDD in westernized countries has been increasing. There are many arguments as to why this is occurring, but one compelling case which has been made in various ways by Allen Frances (Frances, 2013), Nassir Ghaemi (Ghaemi, 2013), Jerome Wakefield (Horowitz \& Wakefield, 2007), David Goldberg (Goldberg, 2011) and others involves questioning the diagnostic category itself. The DSM-as an operationally-based set of symptom clusters designed to achieve reliability (if not validity) in diagnosis-has been criticized and politicized in many ways over the years as a psychiatric tool. But the fact remains that the 157 diagnoses contained in the DSM are not equally biologically valid. MDD, for all its ubiquity and attention, is heterogeneous as a category and is often applied to forms of human suffering that occur on a normal continuum. Such forms of human suffering are real and nontrivial, however current psychiatric screening practices (including those used in the Carhart-Harris paper) likely conflate them with true pathological depression, which limits the signal that can be detected from an intervention meant to treat the latter. The pragmatic challenge of requisite serotonergic antidepressant discontinuation prior to classic psychedelic interventions also impacts this set of issues. This not only introduces a selection bias towards less severe cases (where the number needed to treat would increase and there is higher biological heterogeneity of illness) but disadvantages psychedelic interventions if there is an additional overlay of SRI withdrawal phenomena. The Imperial College trial required subjects to be off serotonergic antidepressants for two weeks, however there are reasons to 
think this is an insufficient amount of time for normalization of 5HT2A receptor density and hence possible blunting of psychedelic effect.

\section{MEASURING DEPRESSION: LIMITATIONS OF CURRENT RATING SCALES}

The evaluation and diagnosis of MDD cannot be clearly tied to an observable and measurable bodily function (in contrast to other medical conditions such as, say, hypertension). In this way, MDD exemplifies what we might think of as an 'inferred variable' (McClintock, Haley, \& Bernstein, 2011). As such, the magnitude of a patient's depression is an inference based on a combination of signs and symptoms, such as sleep, subjective sadness, agitation, guilt, loss of interest, weight changes, etc. Different rating scales attempt to characterize MDD severity with differential weighting on these indices. As antidepressant trials have shown, it is possible to have significant changes in, say, HAM-D scores based on simply changes in sleep or appetite: while these symptoms are not necessarily trivial they are not necessarily the primary targets we think of with 'antidepressant' treatment and do not necessarily align with patients' primary motivations for seeking treatment. In this sense, medications that are sedating, or that increase appetite, have a significant advantage in clinical trials despite perhaps not being superior in terms of the outcomes we truly want to achieve for a depressed patient. These less-central aspects to a depressive state are more likely affected by standard daily treatments than by 1-2 psilocybin sessions, possibly advantaging those interventions insofar as these outcome measures. While patient-report scales such as the QIDS can perhaps better capture some of the subjective changes that are important to the individual, currently used rating scales for MDD do no adequately account for functional outcomes, quality of life, sense of well-being, or sense of meaning: outcomes that are phenomenologically significant for remission of a depressive episode and also seemingly robustly affected by psychedelic experience.

\section{SYMPTOM MITIGATION VS. SPIRITUAL GROWTH}

The third reason that MDD is a problematic target for early psychedelic science is that there are inherent conflicts between psychiatric conceptualizations of symptom reduction in MDD and the perspectival shifts on one's own suffering that are frequently occasioned by psychedelic experience. The extent to which this is a problem is an empirical question: it may be the case that the insights and perspective changes that can be catalyzed by psychedelic experience-in particular mystical-type experiences-are directly translatable to reductions on symptom subscales. And there seem to be reasons to think that there is at least overlap here. However there are reasons to have hesitations about this proposition. Modern psychiatric pharmacological approaches to MDD, centered around standard antidepressant interventions, carry an implicit set of assumptions regarding human suffering and its amelioration. Symptoms represent a disease state and are directly targeted with a goal of remission: one should be feeling other than one is. This is a framework tacitly communicated to patients in the context of antidepressant treatment, along with other naively dualistic conceptions of MDD being better explained as a 'brain disease' than a more complex set of emotional, psychologi$\mathrm{cal}$, and physiological responses to a set of stressors or life circumstances that exceed capacity for resiliency. Compare this with the frequently endorsed psychedelic-catalyzed insight of acceptance: that things are exactly as they are, that one is a part of this larger fabric, and that-at some rockbottom level-everything is $O K$. Now, it is certainly conceivable-and perhaps even likely in many circumstances-that this kind of experientially-based existential insight has notable downstream effects on one's longitudinal mood. However, it is entirely consistent that one can have this experience, feel notably changed in a meaningfully positive and sustained way, and continue to experience similar difficulties. Psychedelics have been employed as tools for spiritual growth for millenia. While there is a diversity of spiritual frameworks that have been brought to bear on the psychedelic experience that represent a range of commitments, it seems fair to say that the path of spiritual growth, whether that is understood in contemplative traditions or shamanic traditions, is non-linear, paradoxical, and in many ways orthogonal to what might be captured on, say, the HAM-D. While a repeated finding in clinical trials with psilocybin has been that the magnitude of therapeutic change is predicated on degree of mystical experience attained the nature of this relationship remains unclear and incompletely characterized and there is at least some recent evidence to suggest that antidepressant effects of psilocybin are not mediated by serotonin $2 \mathrm{~A}$ agonism (which is directly linked to experiential aspects of psychedelics) (Hesselgrave, Troppoli, Wulff, Cole, \& Thompson, 2021). Ketamine within certain dosage ranges- can also reliably occasion mystical-type experiences however the duration of antidepressant effect is seemingly short-lived in a way that cannot be fully accounted for by relying on experiential levels of explanation. Given uncertainty as to these various causal levels, it would be reasonable at this stage of research to disentangle these explanations, bracket speculations on the role of 'mystical experience,' and rely primarily on clearly measurable behavioral targets for demonstrating effect.

\section{BETTER METHODS AND BETTER TARGETS}

So how ought the research on psychedelic-assisted therapies proceed at present? Again, this is in large part a pragmatic question based on the currently available science. Regardless of the illness category targets, psychedelic science would benefit from improved consistency in methodology. If major depressive disorder is the target, inclusion criteria could be refined to recruit more uniform samples, even within an 
illness category that carries higher heterogeneity. For instance: narrowing to treatment-resistant MDD; exclusion of personality disorders using structured diagnostic interviews monitored by or videotaped by an independent, trained psychiatrist; more consistent utilization of clinicianadministered rating scales (rather than the QIDS); and use of additional rating scales that track items of more importance to the wellbeing of participants (e.g. the McGill Quality of Life Inventory).

However, to maximize chance of demonstrating efficacy within current psychiatric categories of illness, it would make sense to choose conditions with clearly defined behavioral presentations that have high inter-rater reliability and directly observable outcome measures. In this respect, categories such as substance use disorders, eating disorders, somatic symptoms and functional neurological disorders, and obsessive-compulsive disorders offer many advantages. Even categories of 'existential distress' among patients with terminal illness lend themselves to more homogeneity of underlying pathology than MDD, as well as involve consistency among psychological themes amenable to timelimited interventions. Of course, these illness categories also carry their own sets of challenges. For instance, substance use disorders are chronic and difficult to treat with current interventions, as well as heavily influenced by systemic and social factors that are not clearly amenable to individual interventions. Early psychedelic therapy studies showed smaller effect sizes in this study population. Indeed, psychedelic interventions may be found to have less or no effect on more clearly-defined illness categories. However, increased certainty regarding the target condition would at least allow more concrete conclusions to be drawn from the data, including more straightforward accumulation and refinement of empirical evidence for psychedelic-assisted therapies as we develop an understanding of where they are useful, and where they are not.

\section{REFERENCES}

Brody, D. J., \& Gu, Q. (2020). Antidepressant use among adults: United States, 2015-2018. NCHS data brief, no 377. Hyattsville, MD: National Center for Health Statistics.

Carhart-Harris, R.L., Bolstridge, M., Day, C.M.J., Rucker, J., Watts, R., Erritzoe, D. E., et al. (2018). Psilocybin with psychological support for treatment-resistant depression: Six-month followup. Psychopharmacology, 235(2), 399-408.
Carhart-Harris, R., Bolstridge, M., Rucker, J., Day, C., Erritzoe, D., \& Kaelen, M. (2021). Trial of psilocybin versus escitalopram for depression. New England Journal of Medicine, 384(15), 14021411.

Frances, A. (2013). The new crisis of confidence in psychiatric diagnosis. Annals of Internal Medicine, 159(2), 221-222.

Ghaemi, N. (2013). On depression: Drugs, diagnosis and despair in the modern world. Johns Hopkins U Press.

Goldberg, D. (2011). The heterogeneity of "major depression". World Psychiatry, 10(3), 226-228.

Griffiths, R. (2015) Psilocybin, mystical-type experiences, and the treatment of symptoms of anxiety and depression in patients with a life-threatening cancer diagnosis. Presentation at the 168th Annual Meeting of the American Psychiatric Association, Abstract 1162.

Grob, C. S., Bossis, A. P., \& Griffiths, R. R. (2013). Use of the classical hallucinogen psilocybin for treatment of existential distress associated.

Hesselgrave, N., Troppoli, T., Wulff, A., Cole, A., \& Thompson, S. (2021). Harnessing psilocybin: Antidepressant-like behavioral and synaptic actions of psilocybin are independent of 5-HT2R activation in mice. Proceedings of the National Academy of Sciences, 118(17), e2022489118. https://doi.org/10.1073/pnas. 2022489118.

Horowitz, A., \& Wakefield, J. (2007). The loss of sadness: How psychiatry transformed normal sorrow into depressive disorder. Oxford U Press.

Howland, R. H. (2008). Sequenced treatment alternatives to relieve depression (STAR* D)-Part 2: Study outcomes. Journal of Psychosocial Nursing and Mental Health Services, 46(10), 21-24.

Jakobsen, J., Gluud, C., \& Kirsch, I. (2020). Should antidepressants be used for major depressive disorder? BMJ, 25(4).

Johnson, M., Garcia-Romeu, A., Cosimano, M., \& Griffiths, R. (2014). Pilot study of the 5- HT2AR agonist psilocybin in the treatment of tobacco addiction. Journal of Psychopharmacology, 28(11), 983-992.

Johnson, M., Garcia-Romeu, A., \& Griffiths, R. (2017). Long-term follow-up of psilocybin- facilitated smoking cessation. The American Journal of Drug and Alcohol Abuse, 43(1), 55-60.

Kirsch, I. (2019). Placebo effect in the treatment of depression and anxiety. Frontiers in Psychiatry, 10, 407.

McClintock, S. M., Haley, C., \& Bernstein, I. H. (2011). Psychometric considerations of depression symptom rating scales. Neuropsychiatry, 1(6), 611.

National Institutes of Health. U.S. National Library of Medicine. Clinicaltrials.gov. https://clinicalttrials.gov/ct2/results? cond $=\&$ term $=$ psilocybin $\&$ cntry $=\&$ state $=\&$ city $=\&$ dist $=$ [Accessed 29th April 2021]. 